Check for updates

Cite this: RSC Adv., 2017, 7, 23942

Received 19th March 2017

Accepted 21st April 2017

DOI: 10.1039/c7ra03211k

rsc.li/rsc-advances

\section{In vitro-in vivo evaluation of hyaluronic acid-based amphiphilic copolymers for tumour targeted delivery: the role of hydrophobic groups $\uparrow$}

\author{
Zhihong Zhu, Dongyang Li, Yuenan Li, Xinggang Yang* and Weisan Pan (D)*
}

Polymeric micelles are widely used as suitable nano-carriers for a variety of therapeutic applications. Exploring the role of hydrophobic groups on the properties of the amphiphilic carrier and micelles were the aim of this study. Using hyaluronic acid (HA), cholesterol (CHOL) and octadecanoic acid (OA), two amphiphilic polymers were prepared and fully characterized by ${ }^{1} \mathrm{H}$ NMR, FTIR and DSC. Compared with HA-SA-CYS-OA, HA-SA-CYS-CHOL had a lower critical micellar concentration (CMC) producing docetaxel (DTX)-loaded micelles of a smaller particle size, higher encapsulation efficiency (EE) and drug loading (DL\%). However, HA-SA-CYS-OA had the greater EE when loaded with coumarin-6 (Cou6), suggesting that hydrophobic groups had a degree of selectivity for drugs. Cellular uptake demonstrated that the two micelles clearly increased the internalization of DTX via CD44 receptor-mediated endocytosis. In vitro cytotoxic experiments showed that HA-SA-CYS-OA micelles were generally more cytotoxic to MCF-7, A549 and S-180 cells on account of the toxicity of octadecanoic acid while in vivo animal experiments confirmed that $\mathrm{HA}-\mathrm{SA}-\mathrm{CYS}-\mathrm{CHOL}$ micelles exhibited better tumor-targeting properties and antitumor effects. From these preliminary evaluations, it is possible to conclude that hydrophobic groups not only affect the stability and drug-loading capacity of the amphiphilic carriers, but also influence the distribution, metabolism, excretion and ultimately, the antitumor efficacy of micelles.

\section{Introduction}

Polymeric micelles, with a great difference in hydrophilic properties between the hydrophilic and the hydrophobic groups, are widely used as suitable nano-carriers for a variety of therapeutic applications. ${ }^{1}$ It has been reported that the distribution of micelles in the body may be determined mainly by their size and surface properties ${ }^{2}$ and the physicochemical and biological properties of the micelles are mainly determined by the functionalization of the outer surface. Based on this, a number of passive and active targeting polymeric micelles have been developed. However, the anti-tumour effect in the clinic is not significant. As an essential component of an amphiphilic copolymer, the physical and chemical properties of hydrophobic groups can influence the association behaviour of micelles in solution and their affinity for interfaces. ${ }^{3}$ Therefore, we hypothesize that the hydrophobic groups have an effect on the pharmacokinetic and physicochemical properties of the micelles. It is time to take hydrophobic groups into account for developing better antitumour drug delivery systems. Studies have shown that hydrophobic groups can increase the solubility of hydrophobic drugs

Shenyang Pharmaceutical University, No. 103, Wenhua Road, Shenyang 110016, China.E-mail:pppwwwsss@163.com; yangxg123@163.com

$\dagger$ Electronic supplementary information (ESI) available. See DOI: $10.1039 / \mathrm{c} 7 \mathrm{ra} 03211 \mathrm{k}$ and the stability of the micelles. ${ }^{4,5}$ It also has been reported that the hydrophobic groups can affected the rheological properties of the carrier ${ }^{6}$ and the antibacterial activity. ${ }^{7}$ Even more the length of hydrophobic groups displayed the decisive effect on the gelation behaviour. ${ }^{8}$ However, researchers have not systematically and holistically studied the influence of hydrophobic groups on the properties of the amphiphilic carrier and micelles yet. In order to fill this gap, our study focuses primarily on the role of the different hydrophobic groups on the anti-tumour effects and on the properties of the amphiphilic carrier and micelles.

CD44 is a widely distributed cell surface type I transmembrane glycoprotein, the over-expression of which is connected to the motility, survival and proliferation of the tumour cell and the formation of the tumour microenvironment. Studies have shown that tumour cells often express a number of CD44 variants, particularly when the cancer is in an advanced stage. ${ }^{9} \mathrm{CD} 44$, especially CD44v isoforms, have been identified as CSC surface markers in different types of cancers, ${ }^{10}$ making $\mathrm{CD} 44$ a potential target of tumour therapy. The N-terminal part of CD44 enables binding to hyaluronic acid (HA), ${ }^{11}$ a kind of acid mucopolysaccharide composing of repeated disaccharide units of D-glucuronic acid and $N$-acetyl glucosamine. ${ }^{12} \mathrm{HA}$, with functional groups of carboxylic acid and hydroxyl moieties can be chemically modified by crosslinking or conjugation reactions. ${ }^{13}$ In addition, HA is an important component of the extracellular matrix and HA modified polymer is a biodegradable, biocompatible, nontoxic and non- 
immunogenic. There have been emerged more and more nanoplatforms mainly with hyaluronic acid and other hydrophobic groups, such as octenyl succinic anhydride (OSA), ${ }^{14}$ deoxycholic acid (DOCA), ${ }^{15}$ and octadecylamine. ${ }^{16}$ Cholesterol is a vital component of cell membranes accounting for over $20 \%$ of the plasma membrane lipids and, due to its biocompatibility and biodegradability, cholesterol is often used in studies of drug delivery systems involving nanoparticles. ${ }^{17,18}$ Modification with cholesterol results in significant improvement of the cellular uptake of polymers for drug delivery applications. ${ }^{19}$ Octadecanoic acid (OA) is a hydrophobic saturated fatty acid which has been used as a safe biodegradable dietary additive. OA can be grafted to the hydrophilic group because of its good biosafety and suitable molecular weight. ${ }^{20,21}$ Although cholesterol and OA are widely used in the application of nano-platforms, few studies have involved a comparative investigation of the amphiphilic copolymers composed of octadecanoic acid or cholesterol and HA and, so far, few studies have examined whether hydrophobic groups affect the properties of the amphiphilic carrier and anti-tumour effect of micelles.

Here, our goal was to synthesize two kinds of amphiphilic carriers with the same hydrophilic group and different hydrophobic groups to explore whether the different hydrophobic groups have effects on the nature of the amphiphilic carrier and micelles and even the anti-tumour effect of micelles.

\section{Experimental section}

\section{Materials}

HA with an average molecular weight of $44 \mathrm{kDa}$ was provided by Shandong Freda Biopharm Co., Ltd. (Shandong, China).
Triethylamine, octadecanoic acid (OA, 99\%), succinic anhydride (SA, 99\%) was obtained from YUWAN GROUP (Shandong, China). $\mathrm{N}$-Hydroxysuccinimide (NHS, 99\%), 4-dimethylaminopyridine (DMAP, 99\%), benzotriazole-1-yl-oxytripyrrolidinophosphonium hexafluorophosphate (PyBOP), 1-(3-dimethylaminopropyl)-3ethylcarbodiimidehydrochloride (EDCI, 99\%) was obtained from shanghai Medpep Co., Ltd. (Shanghai, China). 3-(4,5-Dimethylthiazol-2-yl)-2,5-diphenyl-tetrazoliumbromide (MTT, 98\%) and cholesteryl chloroformate (98\%) were obtained from Alfa Aesar (China) Chemical Co., Ltd. (Shanghai, China). DiR was obtained from Beijing Fanbo Biochemicals Co., Ltd. (Beijing, China). Docetaxel (DTX) was provided by Jiangsu Hengrui Pharmaceutical Co. Ltd. (Jiangsu, China). Cystamine dihydrochloride (CYS, 98\%) was obtained from J\&K Scientific Ltd., (Beijing, China). Commercial docetaxel solution (Taxotere $®$ ) was obtained from SanofiAventis (France). S-180 murine sarcoma cells, A549 non-small lung cancer cells and MCF-7 human breast cancer cells were purchased from Type Culture Collection of Chinese Academy of Sciences (Shanghai, China). Dulbecco's Modified Eagle Medium (DMEM) and Roswell Park Memorial Institute (RPMI) 1640 medium were obtained from Hyclone Co., Ltd. (Thermo Fisher Scientific., UK) and fetal bovine serum (FBS) were obtained from Sijiqing Co., Ltd. (Hangzhou, China). All other chemicals and reagents used were of analytical grade or cell culture grade and obtained commercially.

\section{Synthesis of copolymers}

Synthesis of succinic anhydride (SA)-modified HA (HA-SA). HA-SA was synthesized as described in the literature. ${ }^{14} \mathrm{HA}$ $(0.509 \mathrm{~g})$ was dissolved in $40 \mathrm{~mL}$ formamide containing 4dimethylaminopyridine (DMAP) $(0.090 \mathrm{~g}, 0.5 \mathrm{mmol})$ and

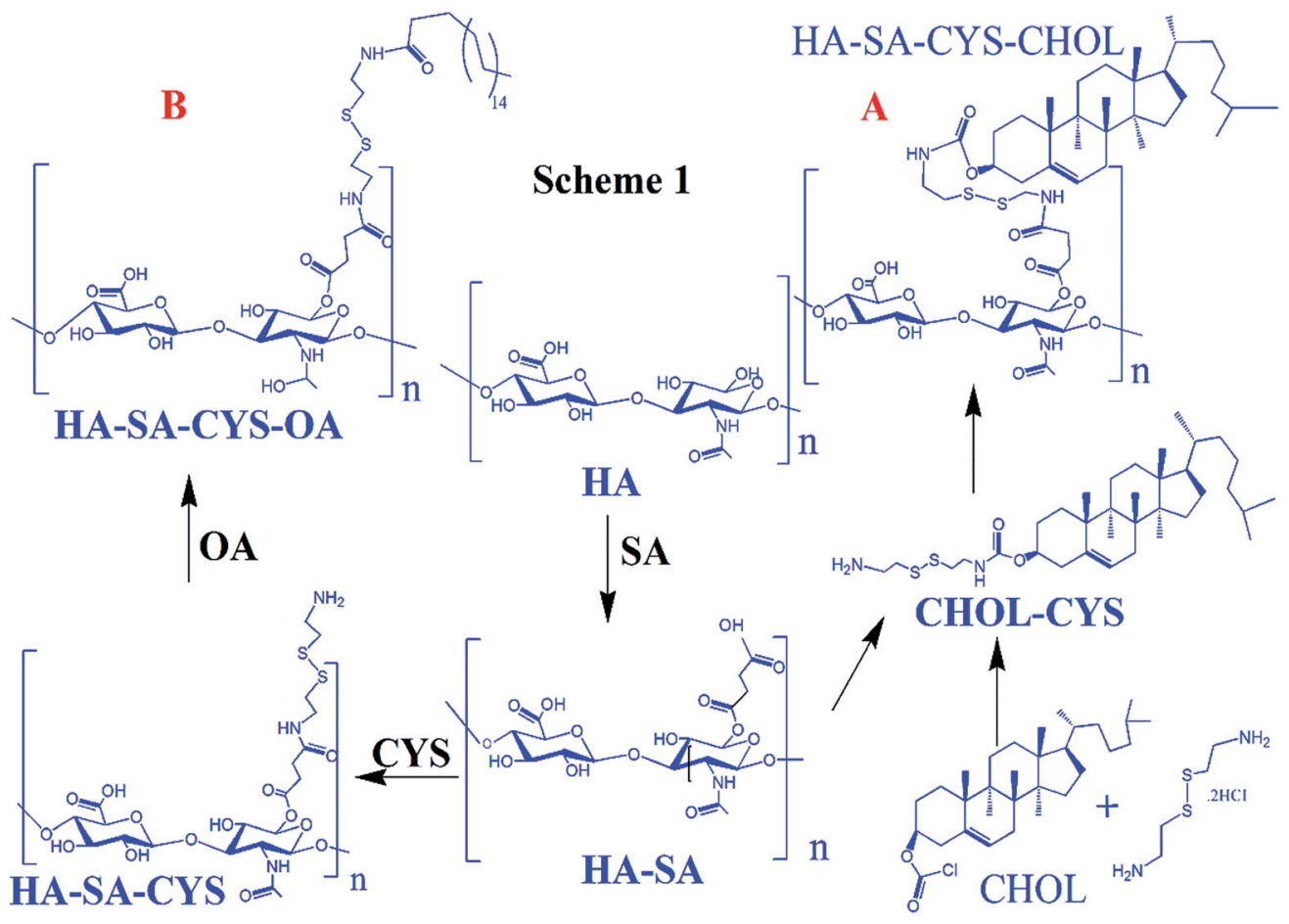

Scheme 1 Synthesis scheme HA-SA-CYS-CHOL (A), HA-SA-CYS-OA (B). 
triethylamine $(4.5 \mathrm{~mL})$. Then, succinic anhydride $(0.506 \mathrm{~g}, 0.5$ $\mathrm{mmol}$ ) in $10 \mathrm{~mL}$ formamide was dropwise added into HA solution and stirred for one and a half days, dialyzed against an excess of water in semipermeable tubes (MWCO 8-14 kDa) for three days. The final product was lyophilized. The product, HASA, was finally obtained after lyophilization as a white solid (0.76 g, yield: $72.4 \%$ ).

Synthesis of octadecanoic acid (OA)-modified HA-SA (HASA-CYS-OA)

Synthesis of HA-SA-CYS. HA-SA-CYS was obtained by coupling cystamine (CYS) to HA-SA by formation of an amide. ${ }^{22-24}$ In detail, HA-SA (0.4 g) was dissolved in $100 \mathrm{~mL}$ PBS (pH 7.4) with EDCl (0.232 g, $0.12 \mathrm{mmol})$, NHS (0.13 g, 0.12 $\mathrm{mmol}$ ) and the mixture was stirred at $25{ }^{\circ} \mathrm{C}$ for $30 \mathrm{~min}$. Then CYS (2.25 g, $10 \mathrm{mmol}$ ) was added and, after $5 \mathrm{~h}$, the crude product was dialyzed against water in semipermeable tubes (MWCO 8-14 kDa) for three days to obtain the HA-SA-CYS followed by lyophilization ( $0.19 \mathrm{~g}$, yield: $74.7 \%)$.

Synthesis of HA-SA-CYS-OA. HA-SA-CYS-OA was obtained by conjugating the primary amines of HA-SA-CYS to the free carboxyl groups of OA. ${ }^{23,25}$ Firstly, OA (0.36 g, $\left.1.25 \mathrm{mmol}\right)$ was dissolved in $10 \mathrm{~mL}$ DMSO with PyBOP (0.78 g, $1.5 \mathrm{mmol})$. Then,

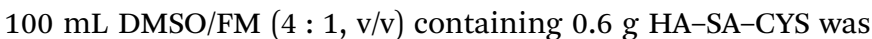
dropwise to OA solution for reaction for two days, and then the resulting solution was dialyzed against an excess of water for 3 days, and lyophilized, stored at $-20{ }^{\circ} \mathrm{C}(0.49 \mathrm{~g}$, yield: $51 \%)$. The whole process is shown in Scheme 1A.

Synthesis of cholesterol (CHOL)-modified HA-SA (HA-SACYS-CHOL)

Synthesis of the CHOL-CYS. ${ }^{26}$ The CHOL-CYS was synthesized as previously described by the acylation of one of the amine groups of cystamine dihydrochloride. Cystamine dihydrochloride $(0.113 \mathrm{~g}, 0.5 \mathrm{mmol})$ was dissolved in a mixture of $5 \mathrm{~mL} 3.5 \mathrm{M}$ $\mathrm{NaOH}$ and $4 \mathrm{~mL}$ chloroform. This solution was heated to $50{ }^{\circ} \mathrm{C}$, and $10 \mathrm{~mL}$ chloroform containing cholesteryl chloroformate $(0.225 \mathrm{~g}, 0.5 \mathrm{~mol})$ was added dropwise while stirring. After $2 \mathrm{~h}$, the aqueous phase was discarded. The remaining organic phase was extracted three times with distilled water, saturated $\mathrm{NH}_{4} \mathrm{Cl}$ and saturated $\mathrm{NaCl}$, respectively. The organic layer was evaporated to dryness, by vacuum drying $(0.31 \mathrm{~g}, 91.2 \%)$. The molecular weight of CHOL-CYS determined by ESI-MS (Bruker Customer, micrOTOF-Q 125) and $m / z 564.4\left(\mathbf{M}^{+}\right)$(Fig. S6 $\dagger$ ).

Synthesis of $\mathrm{HA}-\mathrm{SA}-\mathrm{CYS}-\mathrm{CHOL}{ }^{19}{ }^{\mathrm{HA}-\mathrm{SA}-\mathrm{CYS}-\mathrm{CHOL} \text { was }}$ synthesized by activation of HA-SA $(0.5 \mathrm{~g}, 1.24 \mathrm{mmol}$ carboxyl groups) in $100 \mathrm{~mL}$ DMSO/FM ( $4: 1, \mathrm{v} / \mathrm{v})$ with PyBOP $(0.78 \mathrm{~g}, 1.5$ mmol) and DMAP $(0.24 \mathrm{~g}, 2 \mathrm{mmol})$ under continuous stirring. Then, CHOL-CYS (0.435 g, $0.77 \mathrm{mmol})$ in $15 \mathrm{~mL}$ DCM was added to react for 2 days at $25{ }^{\circ} \mathrm{C}$. The product was dialyzed in semipermeable tubes (MWCO 8-14 kDa) against water for three days, then lyophilized and stored at $-20{ }^{\circ} \mathrm{C}(0.46 \mathrm{~g}, 49.2 \%)$. The whole process is shown in Scheme $1 \mathrm{~B}$.

\section{Characterization of copolymers}

${ }^{1} \mathbf{H}$ nuclear magnetic resonance spectroscopy (NMR). The chemical structures of the copolymers were confirmed by ${ }^{1} \mathrm{H}$ NMR spectra using a $400 \mathrm{MHz}$ NMR spectrometer (Bruker,
Switzerland). $\mathrm{D}_{2} \mathrm{O}$ or $\mathrm{CDCl}_{3}$ was used as the nuclear magnetism reagent. The degree of substitution (DS) which is defined as the number of cholesterol or octadecanoic acid molecules per 100 sugar residues of HA was also determined using ${ }^{1} \mathrm{H}$ NMR. ${ }^{\mathbf{8 1 9 , 2 7 , 2 8}}$ The integrated signals for cholesterol methylene group (2.29 ppm), $N$-acetyl group of HA (1.96 ppm), and octadecanoic acid methylene group (1.24 ppm) have been selected for the calculation of DS of cholesterol and octadecanoic acid.

Fourier transform infrared (FTIR). Fourier transform infrared (FTIR) was performed using a FTIR spectrometer (Bruker IFS55, Germany) over the range of $4000-500 \mathrm{~cm}^{-1}$ with a resolution of $4 \mathrm{~cm}^{-1}$. KBr disks was used to prepare sample (1 mg sample in $200 \mathrm{mg} \mathrm{KBr}) .^{29}$

Differential scanning calorimetry (DSC). DSC analysis was performed on a TA-60 WS DSC-60 differential scanning calorimetry (Shimadzu, Japan). Samples were heated from $40{ }^{\circ} \mathrm{C}$ to $250{ }^{\circ} \mathrm{C}$ with a heating rate of $10{ }^{\circ} \mathrm{C} \mathrm{min}^{-1}$. $^{30}$

Gel permeation chromatography (GPC). The MWs and the MWDs of HA-SA-CYS-CHOL and HA-SA-CYS-OA copolymer were examined by gel permeation chromatography (GPC) using a single TSKgel GMPWXL gel column (TSK, Japan). Deionized water containing $0.1 \mathrm{~N} \mathrm{NaN}_{3}$ and $0.06 \% \mathrm{NaN}_{3}$ was the mobile phase and was pumped with a Shimadzu LC20 pump at a flow rate of $0.6 \mathrm{~mL} \mathrm{~min}^{-1}$. A Shimadzu RID-20 differential refractometer was used to measure the refractive index signal. The calibration curve was based on narrow $M_{\mathrm{w}}$ linear poly(ethylene oxide).

Measurement of critical micellar concentration (CMC). Pyrene was used as a fluorescence probe to confirm the micelle forming ability of HA-SA-CYS-CHOL and HA-SA-CYS-OA conjugates. ${ }^{16,30}$ In detail, pyrene $(1.238 \mathrm{mg})$ was dissolved in 100 $\mathrm{mL}$ acetone, added to $100 \mathrm{~mL}$ volumetric flasks and dried. Different concentrations of HA-SA-CYS-CHOL and HA-SACYS-OA were added to the volumetric flasks. The final concentration of pyrene was $6.0 \times 10^{-7} \mathrm{M}$. The volumetric flasks were sonicated for $30 \mathrm{~min}$ in a water bath and then kept $40{ }^{\circ} \mathrm{C}$ for $12 \mathrm{~h}$. The fluorescence spectrum of each solution was recorded on a BioRed microplate reader (Model500, USA). The intensity ratio of the first peak $\left(I_{1}, 373 \mathrm{~nm}\right)$ to the third peak $\left(I_{3}\right.$, $384 \mathrm{~nm}$ ) was used for the calculation of CMC.

Preparation and characterization of DTX-loaded micelles. A modified emulsification solvent evaporation method was used to prepare the DTX-loaded HA-SA-CYS-CHOL and HA-SA-CYS-OA micelles. ${ }^{22,31}$ Briefly, $10 \mathrm{mg}$ HA-SA-CYS-CHOL or HA-SA-CYSOA copolymer was dispersed in $5 \mathrm{~mL}$ distilled water. Then, $0.25 \mathrm{mg}$ DTX in $0.5 \mathrm{~mL}$ ethanol was added followed by sonication at $300 \mathrm{~W}$ for $10 \mathrm{~min}$ in an ice bath with a probe-type ultrasonicator (SJIA-950W; Ningbo Yin Zhou Sjia Lab Equipment Co., Ltd, Ningbo, China). Then stirred for $12 \mathrm{~h}$ at $25{ }^{\circ} \mathrm{C}$, centrifuged at $3000 \mathrm{rpm}$ for $15 \mathrm{~min}$, passed through $0.45 \mathrm{~mm}$ pore size filter membrane, and the filtrate was stored at $4{ }^{\circ} \mathrm{C}$ until required. The blue lipophilic fluorescent dye DiR and green lipophilic fluorescent dye Cou6 were chosen to be the fluorescent probe and prepared by the same procedure like DTX.

The shape and morphology of the nanoparticles prepared using HA-SA-CYS-CHOL or HA-SA-CYS-OA copolymer were 
examined by transmission electron microscopy (TEM) (JEM$1200 \mathrm{EX}(120 \mathrm{kV})$, JEOL, Japan) after negative staining.

The average particle size (diameter, nm), polydispersity index (PDI) and zeta potential of the HA-SA-CYS-CHOL and HA-SA-CYS-OA micelles were measured by dynamic light scattering (DLS) using a Zetasizer Nano ZS90 instrument (Malvern, UK) with a scattering angle of $90^{\circ}$ at $25^{\circ} \mathrm{C}$.

DSC was used to measure the physical or chemical changes of micelles with the change of temperature.

The changes in crystalline state of drug was analysed by Powder X-ray diffraction analysis (PXRD) used a D8 ADVANCE powder diffraction meter (Bruker, AXS, Germany) with a scanning angular scope of $5-50^{\circ}(2 \theta)$ in scanning speed of $6^{\circ}$ per minute.

The encapsulation efficiency (EE\%) and drug loading (DL\%) was determined by HPLC. Briefly, $0.1 \mathrm{~mL}$ samples of micelles were disrupted with $9.9 \mathrm{~mL}$ acetonitrile followed by sonication disruption for $10 \mathrm{~min}$ and centrifugation for $10 \mathrm{~min}$ at $10000 \mathrm{rpm}$. HPLC conditions were as follows: a Neptune C18 (Neptune C18 5u, Nu-Analytical, CA, USA) was used at a flow rate

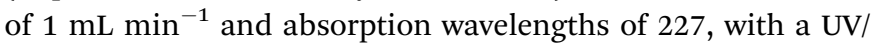
VIS detector. The mobile phase used for docetaxel was acetonitrile/water $\left(3: 2\right.$ volume ratio). ${ }^{31-33}$ The EE and DL were calculated using the following formulas:

$$
\text { DL }(\%)=(\text { amount of drug in NPs }) /(\text { amount of NPs }) \times 100
$$

$\mathrm{EE}(\%)=($ amount of drug in NPs)/(amount of the feeding drug) $\times 100$

In vitro drug release assay. The in vitro drug release of DTXloaded micelles and Taxotere ${ }^{\circledR}$ was experimented in phosphate buffered saline (PBS) of $\mathrm{pH} 7.4 .^{34} \mathrm{In}$ brief, $1 \mathrm{~mL}$ of each of the different micelles dispersions was sealed in a dialysis bag (MWCO: 8-14 kDa) and immersed into $50 \mathrm{~mL}$ PBS containing $0.25 \%$ Tween $80 .^{35}$ The released solution was kept at $37{ }^{\circ} \mathrm{C}$ with shaking $(100 \mathrm{rpm})$. At pre-determined times; $1 \mathrm{~mL}$ release medium was withdrawn and replaced with an equivalent volume of fresh medium. The withdrawn medium was centrifuged at $10000 \mathrm{rpm}$ for $5 \mathrm{~min}$ and assayed by HPLC.

\section{In vitro cell study}

In vitro cytotoxicity. The cytotoxicity of blank, DTX-loaded HA-SA-CYS-CHOL, HA-SA-CYS-OA micelles and Taxotere ${ }^{\circledR}$ against MCF-7, A549 and S-180 cells was evaluated using a standard MTT assay. ${ }^{33,36}$ In detail, cells were seeded at a density of $1 \times 10^{4}$ cells per well in 96-well plates and incubated for $24 \mathrm{~h}$ to allow cell attachment (for S-180 cells, the drug was added directly to the 96-well plates). Taxotere $₫$, blank micelles and DTX-loaded micelles in a concentration gradient were treated with these cells at $37^{\circ} \mathrm{C}$. After $24 \mathrm{~h}$ incubation, the cells were centrifuged, the medium were discarded and replaced with $100 \mu \mathrm{L}$ fresh medium, $10 \mu \mathrm{L}$ MTT $\left(5 \mathrm{mg} \mathrm{mL}^{-1}\right)$ was added and then the solutions were further incubated in a $\mathrm{CO}_{2}$ incubator for $4 \mathrm{~h}$. The generated formazan was solubilized with $100 \mu \mathrm{L} 10 \% \mathrm{SDS}, 0.01 \mathrm{M} \mathrm{HCl}$ solution. The plates were kept in dark for $12 \mathrm{~h}$ for dissolution of the formed formazan crystals. The absorbance at $570 \mathrm{~nm}$ was recorded using a BioRed microplate reader (MK3, Thermo, Massachusetts, USA). Each drug concentration measurement was the mean value of eight wells. The relative cell viability (\%) was calculated using the following equation:

$$
\text { Cell viability }(\%)=\left(A_{\text {sample }}-A_{\text {blank }}\right) /\left(A_{\text {control }}-A_{\text {blank }}\right) \times 100
$$

where $A_{\text {control }}$ and $A_{\text {sample }}$ are the absorbance in the absence and in the presence of sample treatment, respectively. $A_{\text {blank }}$ is the absorbance of the medium. Also, the growth inhibition concentration for $50 \%$ of the cell population $\left(\mathrm{IC}_{50}\right)$ was calculated using Compusyn1.0 software (http://www.combosyn.com/ ). ${ }^{37-40}$ All measurements were performed in triplicate.

In vitro cellular uptake. To evaluate the intracellular uptake ability of micelles in MCF-7, A549 and S-180 cells, a fluorescence probe Cou6 $\left(3 \mu \mathrm{g} \mathrm{mL} \mathrm{mL}^{-1}\right)$ was encapsulated in HA-SA-CYSCHOL and HA-SA-CYS-OA micelles. ${ }^{37,41}$ MCF-7, A549 and S-180 cells were seeded in 24 -well plates at a density of $1 \times 10^{5}$ cells per well. After $24 \mathrm{~h}$, the cells were centrifuged, the culture medium was discarded and the cells were incubated with Cou6loaded micelles for $2 \mathrm{~h}$ to examine the cellular uptake. After incubation, the medium was again centrifuged, discarded and the cells were washed with PBS three times. Then, $100 \mu \mathrm{L}$ RIPA cell lysate was added to each well to lyse the cells, and $25 \mu \mathrm{L}$ lysate was used to assay the protein content with a Pierce ${ }^{\circledR}$ BCA Protein Assay Kit (Thermo Scientific, USA). The residue was mixed with $100 \mu \mathrm{L}$ ethanol to extract the Cou6 internalized in the cells and the Cou6 concentration was calculated after ELISA (Model ER-8000, Sanko Junyaku Tokyo, Japan).

Confocal laser scanning microscopy (CLSM) was used to qualitatively examine the intracellular uptake of Cou6 loaded NPs. MCF-7, A549 and S-180 cells were cultured on 6-well plates at a density of $2 \times 10^{5}$ per well for $24 \mathrm{~h}$ (for S-180 cells, poly-L-lysine cover slips were added for adhesion). The medium was then replaced with Cou6-loaded micelles $\left(3 \mu \mathrm{g} \mathrm{mL}{ }^{-1}\right)$. After incubating at $37^{\circ} \mathrm{C}$ for $2 \mathrm{~h}$, the culture medium was removed and the cells were rinsed with PBS three times and fixed with a $4 \%$ paraformaldehyde solution. For nuclear staining, the cells were incubated with 4,6-diamino-2-phenylindole (DAPI) for $5 \mathrm{~min}$ at room temperature, followed by washing with PBS (pH 7.4) three times. The intracellular localization of the nanoparticles was observed using a CLSM instrument (LSM 710, Carl Zeiss, Zeiss Germany).

The cellular uptake mechanism was investigated a competitive inhibition study using free $\mathrm{HA}\left(10 \mathrm{mg} \mathrm{mL}{ }^{-1}\right)$.

In vivo study. Male kunming SPF mice (4-6 weeks old, body wt $20 \pm 2 \mathrm{~g}$ ) were kindly provided by the Experimental Animal Centre of Shenyang Pharmaceutical University. The animals were allowed free access to food and water and allowed to acclimatize for at least 7 days before the experiments. The in vivo animal experiments were conducted under the guidelines approved by the Animal Care Committee at Shenyang Pharmaceutical University and followed the principles of Laboratory Animal Care (People's Republic of China). The tumour xenograft mouse model was established by injecting $1 \times 10^{6} \mathrm{~S}-180$ cells in $200 \mu \mathrm{L}$ physiological saline into the armpit region of the kunming mice. 
In vivo bio-distribution and tumour targeting. Non-invasive near-infrared optical imaging was used to evaluate the biodistribution and tumour-targeting properties of HA-SA-CYSCHOL (OA) micelles in S-180 tumour-bearing kunming mice. The kunming mice with $300-500 \mathrm{~mm}^{3}$ tumours were subjected to NIRF imaging. DiR solution and DiR-loaded HA-SA-CYSCHOL (OA) were injected intravenously into the S-180 mice at a dose of $2.0 \mathrm{mg} \mathrm{DIR} / \mathrm{kg}(n=3)$. At designated times $(1,4,8,12$, and $24 \mathrm{~h}$ ), fluorescent images of the mice were acquired by an in vivo molecular imaging system (Carestream, Rochester, NY, USA) with an excitation and emission wavelength of $720 \mathrm{~nm}$ and $790 \mathrm{~nm}$, respectively. Then the animals were sacrificed and the tumours were excised and examined.

In vivo antitumor efficacy. After approximately 1 week postinoculation, kunming mice with $50-100 \mathrm{~mm}^{3} \mathrm{~S}-180$ tumours were selected for study of the in vivo antitumor efficacy. The S180 mice were randomly divided into 4 groups which received different injections $(n=5)$ as follows: (1) i.v. injection, saline (the control group); (2) i.v. injection, Taxotere ${ }^{\circledR}$ at $2 \mathrm{mg} \mathrm{DTX} / \mathrm{kg}$; (3) i.v. injection, HA-SA-CYS-CHOL micelles at $2 \mathrm{mg}$ DTX/kg; (4) i.v. injection, HA-SA-CYS-OA micelles at $2 \mathrm{mg} \mathrm{DTX} / \mathrm{kg}$; each group was injected every 3 days and the therapeutic effect was evaluated by measuring the tumour volume $(V=(a \times$ $\left.b^{2}\right) / 2, a$ and $b$ are the length and width of the tumour, respectively) and body weight from the first injection. After a 15 day treatment, the mice were sacrificed and their tumours were excised. The obtained tumours were weighed and the tumour inhibition rate (TIR) was calculated using the following formula:

$$
\operatorname{TIR}(\%)=\left(W_{\text {control }}-W_{\text {sample }}\right) / W_{\text {control }} \times 100
$$

Statistical analysis. All data were expressed as mean \pm S.D. Student's two sample $t$-test and one-way ANOVA for multiple groups were used for statistical evaluation. $P<0.05$ was considered statistically significant.

\section{Results and discussion}

\section{Synthesis and characterization of HA-modified copolymers}

The successful synthesis of HA-SA-CYS-CHOL (OA) was confirmed by ${ }^{1} \mathrm{H}-\mathrm{NMR}$ as shown in Fig. 1A. Compared with HA, in the spectrum of HA-SA-CYS-CHOL, there was a characteristic peak at $\delta 2.29\left(-\mathrm{CH}_{2}-\right.$ on $\left.-\mathrm{CH}(\mathrm{O}) \mathrm{CH}_{2}-\right), \delta 5.15(-\mathrm{CH}-$ on cyclohexene) ppm assigned to the steroid rings of the cholesterol fragment, $\delta 2.96\left(-\mathrm{CH}-\right.$ on succinyl $\left.-\mathrm{CO}-\mathrm{CH}_{2}-\right), \delta 2.68$ (-CH- on succinyl-CO- $\mathrm{CH}_{2}-$ ) ppm which belong to the succinyl segment and $\delta 3.17\left(-\mathrm{CH}_{2}-\right.$ on cystamine $\left.-\mathrm{NH}^{-} \mathrm{CH}_{2}-\right), \delta 3.63$ $\left(-\mathrm{CH}_{2}-\right.$ on cystamine-S- $\left.\mathrm{CH}_{2}-\right)$ ppm which belongs to the cystamine dihydrochloride confirmed the formation of HA-SACYS-CHOL conjugates. Similarly, in the spectrum of HA-SACYS-OA, the presence of the characteristic peak at $\delta 1.11\left(-\mathrm{CH}_{2}-\right.$ ), $\delta 1.24\left((\beta) \mathrm{CO}-\mathrm{CH}_{2}-\mathrm{CH}_{2}-\right)$ ppm was assigned to the long chain of the stearate fragment, $\delta 2.71\left(-\mathrm{CH}-\right.$ on succinyl $\left.-\mathrm{CO}-\mathrm{CH}_{2}{ }^{-}\right)$, $\delta 2.77$ (- $\mathrm{CH}$ - on succinyl $-\mathrm{CO}-\mathrm{CH}_{2}-$ ) ppm which belongs to the succinyl segment and $\delta 2.89\left(-\mathrm{CH}_{2}-\right.$ on cystamine $\left.-\mathrm{NH}-\mathrm{CH}_{2}-\right)$, $\delta 3.09\left(-\mathrm{CH}_{2}-\right.$ on cystamine-S- $\left.\mathrm{CH}_{2}-\right)$ ppm which belongs to the cystamine dihydrochloride confirmed the formation of HA-SA-

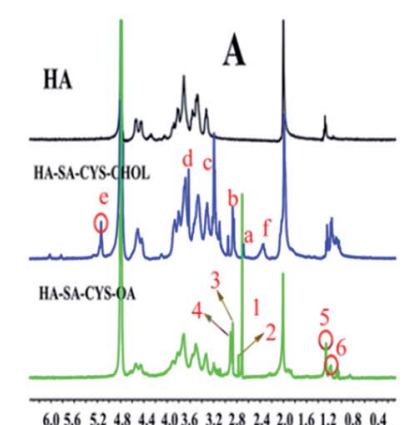

6.05 .65 .24 .84 .44 .03 .63 .22 .82 .42 .01 .61 .20 .80 .4 $\mathrm{ppm}$

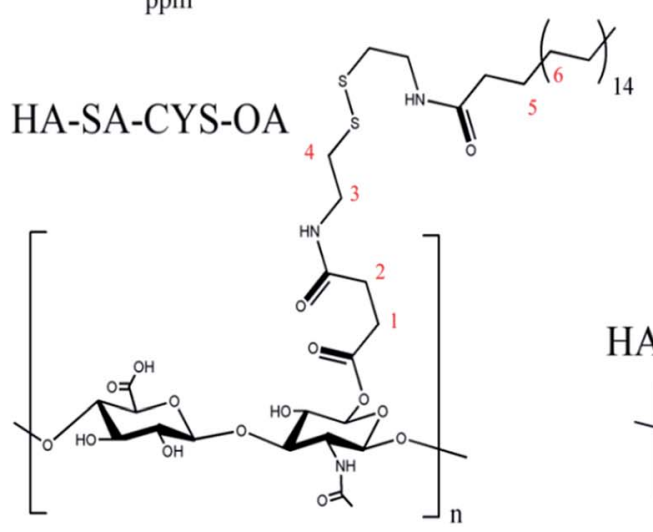

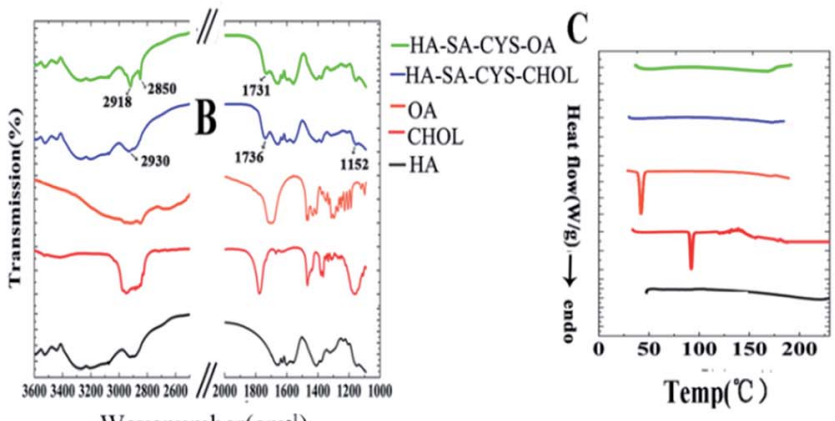

Wavenumber $\left(\mathrm{cm}^{-1}\right)$

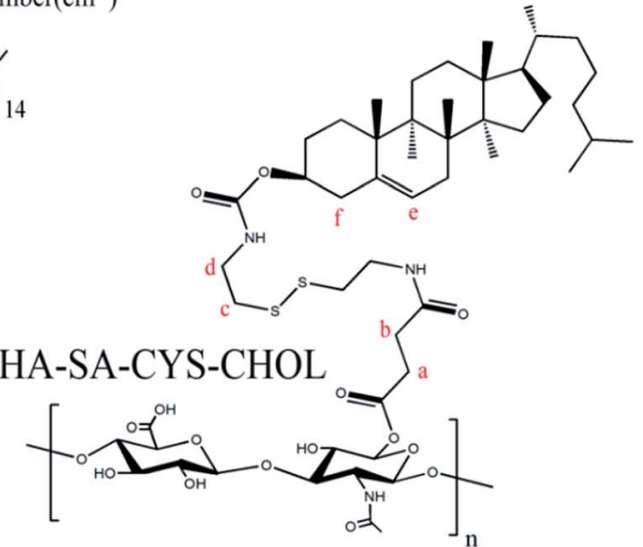

Fig. 1 Images of the ${ }^{1} \mathrm{H} N M R(\mathrm{~A})$ of $\mathrm{HA}, \mathrm{HA}-\mathrm{SA}-\mathrm{CYS}-\mathrm{CHOL}$ and $\mathrm{HA}-\mathrm{SA}-\mathrm{CYS}-\mathrm{OA}, \mathrm{FITR}(\mathrm{B})$, and DSC (C) of HA, CHOL, OA, HA-SA-CYS-CHOL and HA-SA-CYS-OA. 
Table 1 The physicochemical characterization of DTX-loaded self-assembled micelles (mean $\pm \mathrm{SD}, n=3$ )

\begin{tabular}{|c|c|c|c|c|c|c|c|c|c|}
\hline Sample & $\begin{array}{l}\mathrm{DS}^{a} \\
(\%)\end{array}$ & $\begin{array}{l}M_{\mathrm{n}}^{b} \\
\left(\mathrm{~g} \mathrm{~mol}{ }^{-1}\right)\end{array}$ & $M_{\mathrm{w}} / M_{\mathrm{n}}$ & $\begin{array}{l}\mathrm{CMC} \\
\left(\mathrm{mg} \mathrm{mL} \mathrm{mL}^{-1}\right)\end{array}$ & Size (nm) & PDI & Zeta potential (mV) & EE (\%) & DL (\%) \\
\hline HA-SA-CYS-CHOL & 22.5 & 30086 & 1.70 & 0.0079 & $172 \pm 10.2$ & $0.12 \pm 0.004$ & $-32.3 \pm 0.57$ & $89.67 \pm 2.87$ & $4.81 \pm 0.38$ \\
\hline
\end{tabular}

${ }^{a}$ DS of HA-SA-CYS-CHOL and HA-SA-CYS-OA copolymers determined by ${ }^{1}$ NMR. ${ }^{b}$ Molecular weight of HA-SA-CYS-CHOL and HA-SA-CYS-OA copolymers determined by GPC.

CYS-OA conjugates. The DS of HA-SA-CYS-CHOL and HA-SACYS-OA was $22.5 \%$, and $18 \%$ respectively.

FTIR spectra further supported the formation of HA-SACYS-CHOL (OA). As shown Fig. 1B, in the spectra of HA-SACYS-OA, a new band at $1731 \mathrm{~cm}^{-1}$ was assigned to the carbonyl stretching vibration and the peak at $2918 \mathrm{~cm}^{-1}, 2850 \mathrm{~cm}^{-1}$ were due to the symmetrical and asymmetrical stretching vibration of the $-\mathrm{CH}_{2}$ - group. After hydrophobic groups conjugated to the $\mathrm{HA}$, the band at $1152 \mathrm{~cm}^{-1}, 1736 \mathrm{~cm}^{-1}$ and $2930 \mathrm{~cm}^{-1}$, appeared in the spectra of HA-SA-CYS-CHOL, were attributed to the $-\mathrm{C}=\mathrm{C}-\mathrm{H}$, the carbonyl and the $-\mathrm{CH}_{2^{-}}$asymmetrical stretching vibration.

DSC was used to measure the physical or chemical changes of copolymer with the change of temperature. ${ }^{30}$ As shown in Fig. 1C, neither endothermic peak nor exothermic peak could be detected for HA, indicating that HA could maintain the stability of the structure in the experimental temperature. CHOL and OA respectively showed a sharpened endothermic peak at about $120{ }^{\circ} \mathrm{C}$ and $58{ }^{\circ} \mathrm{C}$, illustrating that the two substances are melted at that temperature. When we used chemical methods to link CHOL or OA to HA, we found that the corresponding HA-SA-CYS-CHOL (OA) polymer had no endothermic and exothermic peaks at the experimental temperature, which indicating that the amphiphilic obtained from chemical linking has a good thermal stability in the experimental temperature.

A typical GPC chromatogram is shown in Fig. S5. $\dagger$ As can be seen in Table 1 , the $M_{\mathrm{n}}$ of the HA-SA-CYS-CHOL copolymer was $30086 \mathrm{~g} \mathrm{~mol}^{-1}$, the $M_{\mathrm{w}} / M_{\mathrm{n}}$ was 1.70 , indicating HA-SA-CYSCHOL have broad PDIs. The $M_{\mathrm{n}}$ of the HA-SA-CYS-OA copolymer was $23024 \mathrm{~g} \mathrm{~mol}^{-1}$, the $M_{\mathrm{w}} / M_{\mathrm{n}}$ was 1.07 , indicating a successful living polymerisation. ${ }^{42,43}$

\section{Measurement of critical micellar concentration (CMC)}

The CMC can affect the ability of self-assembly of the amphiphilic copolymer and the structural stability of micelles in vitro and in vivo. ${ }^{44}$ The CMC values of HA-SA-CYS-CHOL and HASA-CYS-OA copolymers were $0.0079 \mathrm{mg} \mathrm{mL}^{-1}$, and $0.0178 \mathrm{mg}$ $\mathrm{mL}^{-1}$, respectively. This low CMC value revealed that the micelles structure can be stable even under extreme dilution, which means that the micelles can be stability in the bloodstream after intravenous administration. Moreover, the HA-SACYS-CHOL copolymer had a lower CMC than the HA-SA-CYSOA copolymer. This may be because cholesterol is more hydrophobic than octadecanoic acid. The greater hydrophobicity drew the formed micelles more compact, and the stronger hydrophobic forces resulted in a lower CMC. Also, more cholesterol was linked to the HA and the CMC of the copolymers reduced as the DS increased. Different hydrophobic groups led to the different DS and CMC values after reacting with HA in the same molar ratio. Although the difference in DS between the two copolymers was not great $(P>0.01)$, the difference in CMC values was highly significant $(P<0.001)$. This proved our hypothesis that the hydrophobic groups affected the nature of the amphiphilic copolymers due to the nature of the hydrophobic groups in itself, such as hydrophobic force and hydrogen bond. Therefore, we further assumed that the hydrophobic groups will affect the properties of the micelles in vitro and in vivo.

\section{Preparation and characterization of DTX-loaded micelles}

For polymeric micelles, the particle size has an important role in the in vivo performance and pharmacokinetics. It has been known that tumour blood vessels are more leaky than normal vessels, and polymeric micelles can leak into the tumour in the range of $200 \mathrm{~nm}$ to $1.2 \mathrm{~mm} .{ }^{45,46}$ As shown in Table 1, the effective diameters of HA-SA-CYS-CHOL and HA-SA-CYS-OA micelles were $172 \mathrm{~nm}$, and $289.3 \mathrm{~nm}$, with a polydispersity index (PDI) of 0.123 , and 0.148 , respectively (Fig. S1-S4†). HA-SACYS-CHOL micelle had a smaller particle size than HA-SACYS-OA. From CMC experiments, we know that compared with HA-SA-CYS-OA, more cholesterol is linked to the HA and cholesterol is more hydrophobic than octadecanoic acid leading HA-SA-CYS-CHOL amphiphilic copolymer with the lower CMC. The particle size decreased with the decrease in the CMC of HA-SA-CYS-CHOL, probably due to the stronger hydrophobic interaction force between DTX and cholesterol core region lead to stronger binding affinity and more compact micellar core packing. ${ }^{16}$

The particle size indicated that the polymeric micelles could traverse tumour vascellum into the tumour stroma and is uptake by the tumour cells but be blocked by normal vessels because of the quite compact endothelial cells. ${ }^{47}$ In addition, both types of micelles had a relatively high negative zeta potentials of around $-33 \mathrm{mV}$ owing to the presence of the ionized carboxyl groups on the HA backbone. The high negatively charged micelles promised an excellent stability in vitro and in vivo owing to the mutually repel, preventing aggregation or precipitation of particles.

As shown in Fig. 2, TEM was performed to visualize directly the size and morphology of DTX-loaded micelles. The spherical shape of the two micelles strongly confirmed a core-shell 

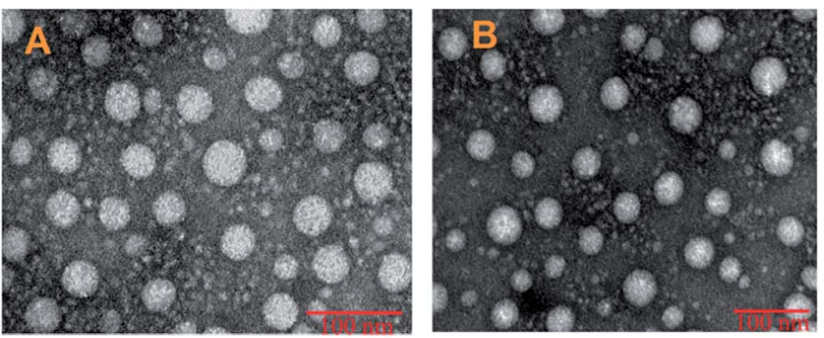

Fig. 2 Transmission electron micrographs of DTX-loaded HA-SACYS-CHOL (A) and HA-SA-CYS-OA (B).

structure with a mean diameter of 50-100 $\mathrm{nm}$ in dehydrated conditions. The size measured by TEM in ultrahigh vacuum conditions was much lower than that measured by DLS in aqueous solution $(172,289.3 \mathrm{~nm})$ which may be due to shrinking of the hydrophilic HA side chain and dehydration of the polymeric main chain during the drying process involved in the preparation of TEM samples. ${ }^{30}$ And DLS analyses the hydrodynamic radius of the dispersed micelles whereas TEM provides the projected surface area based on how much of the incident electrons were transmitted through the sample. So the size measured by TEM is usually smaller than DLS. ${ }^{48}$

The EE and DL values of DTX in HA-SA-CYS-CHOL and HASA-CYS-OA micelles are summarized in Table 1 . When DTX was loaded by these two amphiphilic carriers, much more DTX was encapsulated in HA-SA-CYS-CHOL micelles than HA-SA-CYSOA. The difference between the two amphiphilic carriers was merely the hydrophobic group and HA-SA-CYS-CHOL had a lower CMC than HA-SA-CYS-OA. The DL capacity enhanced with the decrease in the CMC of HA-SA-CYS-CHOL in DTXloaded micelles. ${ }^{16}$ These results could be explained by the fact that the lower the CMC in HA-SA-CYS-CHOL, the stronger hydrophobic interaction force between hydrophobic DTX and the hydrophobic micellar core region lead to the stronger binding affinity. Therefore, we speculate that HA-SA-CYSCHOL has a strong ability to solubilize hydrophobic drugs and the dominant interaction force between the hydrophobic group and the drug is the hydrophobic interaction force. However, when Cou6 was loaded, the HA-SA-CYS-OA packaged more than twice as much Cou6 as HA-SA-CYS-CHOL in the same amount of the carriers $(37.5 \% \pm 3.23$ vs. $7.5 \% \pm 1.35)$, which was the opposite of DTX. Although the hydrophobicity of cholesterol is strong, but the drug encapsulated ability is weaker. Therefore, we speculate that, from the amphiphilic carriers point of view, except the hydrophobic forces, there are other interaction force between the drugs and hydrophobic groups leading to different drug encapsulation abilities, e.g., the spatial 3D configuration of the drug and the hydrophobic groups, the hydrogen bond and so on. It also proved that the amphiphilic copolymer is selective for hydrophobic drugs due to the properties of the hydrophobic groups.

As shown in Fig. 3A and C, free DTX exhibited a single sharp endothermic peak at $223.7{ }^{\circ} \mathrm{C}$, which was melting points of DTX. The blank micelles of HA-SA-CYS-CHOL and HA-SACYS-OA showed no endothermic peak. However, the physical
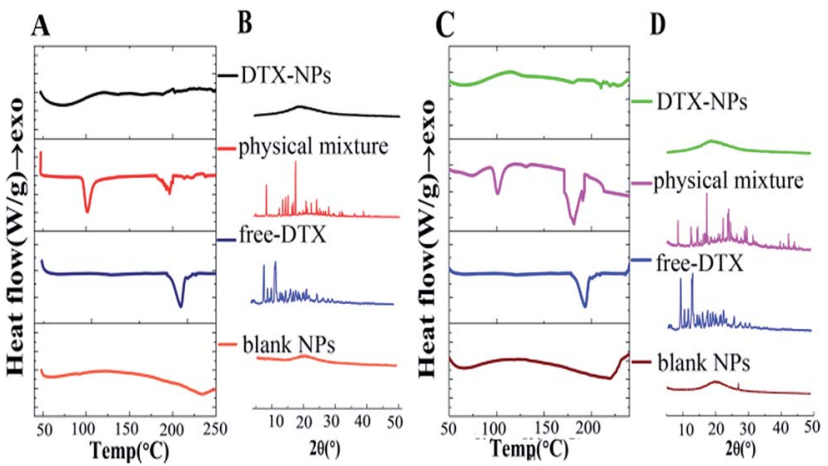

Fig. 3 DSC (A) and XRD (B) of DTX, blank HA-SA-CYS-CHOL micelles, physical mixture of blank HA-SA-CYS-CHOL micelles, DTX and DTX-HA-SA-CYS-CHOL micelles; DSC (C) and XRD (D) of DTX, blank HA-SA-CYS-OA micelles, physical mixture of blank HA-SACYS-OA micelles, DTX and DTX-HA-SA-CYS-OA micelles.

mixture of blank HA-SA-CYS-CHOL micelles and DTX showed a weaker endothermic melting peak at $222.30{ }^{\circ} \mathrm{C}$ and the physical mixture of blank HA-SA-CYS-OA micelles and DTX showed an endothermic melting peak at $223.51^{\circ} \mathrm{C}$.

Nevertheless, the DSC curves of DTX-HA-SA-CYS-CHOL micelles and DTX-HA-SA-CYS-OA micelles demonstrated no melting peaks of DTX, suggesting the drug was entrapped into the micelles in amorphous state. As depicted in Fig. 3B and D, free DTX showed four intense peaks at $2 \theta$ of $8.99^{\circ}, 10.14^{\circ}, 11.25^{\circ}$, $13.94^{\circ}$ and numerous small peaks between $14.57^{\circ}$ and $24.28^{\circ}$. These characteristic XRD peaks of DTX exist in the of physical mixture of blank HA-SA-CYS-CHOL micelles and DTX, blank HA-SA-CYS-OA micelles and DTX, but do not in those of DTXHA-SA-CYS-CHOL micelles and DTX-HA-SA-CYS-OA micelles, suggesting that DTX was either molecularly dispersed in the polymers or distributed in the micelles in an amorphous state. ${ }^{30,49}$

\section{In vitro drug release assay}

Nano-delivery systems are able to control the release of the drug, which greatly reduces the toxicity of the drugs on healthy body tissues. ${ }^{50}$ As shown in Fig. 4, Taxotere ${ }^{\circledR}$ exhibited a $105.09 \%$ release of DTX after $24 \mathrm{~h}$, whereas only $56.88 \%$ of DTX was released from HA-SA-CYS-CHOL micelles, and $63.32 \%$ of DTX from HA-SA-CYS-OA micelles, showing micelles could markedly reduce the drug release in the circulatory system. In addition, compared with HA-SA-CYS-CHOL micelles, much more DTX was released from HA-SA-CYS-OA micelles. The difference between the two amphiphilic carriers was merely the hydrophobic group. The stronger the hydrophobicity of the hydrophobic group, the more strongly the interaction between the hydrophobic group and the drug. Cholesterol has a stronger interaction with DTX with its more hydrophobicity, and DTX can be more stable package in the HA-SA-CYS-CHOL micelles producing a lower drug release. Therefore, we concluded that the hydrophobic groups affected the release of micelles and we also hypothesize that hydrophobic groups will have an influence on the in vivo properties of the micelles. 


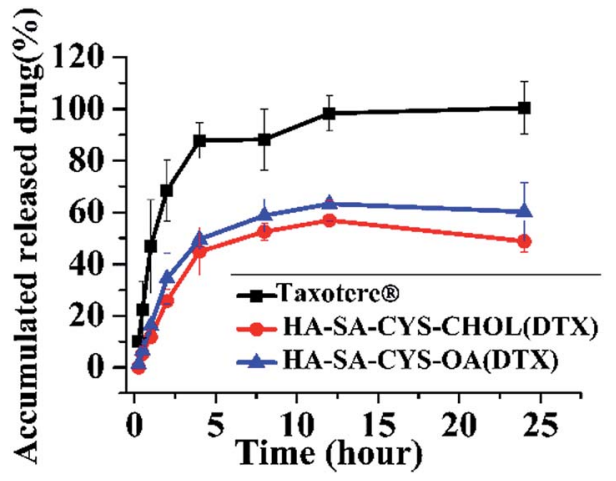

Fig. 4 In vitro release profile of Taxotere ${ }^{\circledR}, \mathrm{HA}-\mathrm{SA}-\mathrm{CYS}-\mathrm{CHOL}$ (DTX) and HA-SA-CYS-OA (DTX) micelles in PBS pH 7.4 at $37^{\circ} \mathrm{C}$. Each data point represents mean $\pm \mathrm{SD}(n=3)$.

\section{In vitro cytotoxicity}

As shown in Fig. 5A-C, blank micelles were slightly toxic to A549 and MCF-7 cells but had little cytotoxicity to S-180 cells. The cytotoxicity of blank micelles may be due to the higher molecular weight fractions of HA showed to inhibit cell cycle progression via a CD44/Rac/ERK pathway ${ }^{51}$ and it was an advantage of using HA-SA-CYA-OA (CHOL) as a drug carrier showed some cytotoxicity. ${ }^{52}$ As shown in Fig. 5D-F and Table 2, DTX-loaded HA-SA-CYS-CHOL and HA-SA-CYS-OA micelles exhibited much higher cytotoxicity than Taxotere ${ }^{\circledR}$. It was reported previously that MCF-7, A549 and S-180 cells were CD44 receptor over-expressing cell lines. ${ }^{53-55}$ Compared with the passive diffusion of free DTX, the higher cytotoxicity of HA-SACYS-CHOL (OA) micelles might be attributed to the higher binding affinity of HA derivatives for CD44 receptors. In addition, HA-SA-CYS-OA micelle was significantly greater toxicity to tumour cells than that of HA-SA-CYS-CHOL in all cell lines. Theoretically, the smaller particle size of micelles could increase the cellular uptake and deliver more DTX into cells, resulting in greater cytotoxicity. However, in our study, HA-SACYS-OA micelles had a greater cytotoxicity with larger particle size. This may be because the hydrophobic group of the two micelles had a greater impact on the cytotoxicity than the
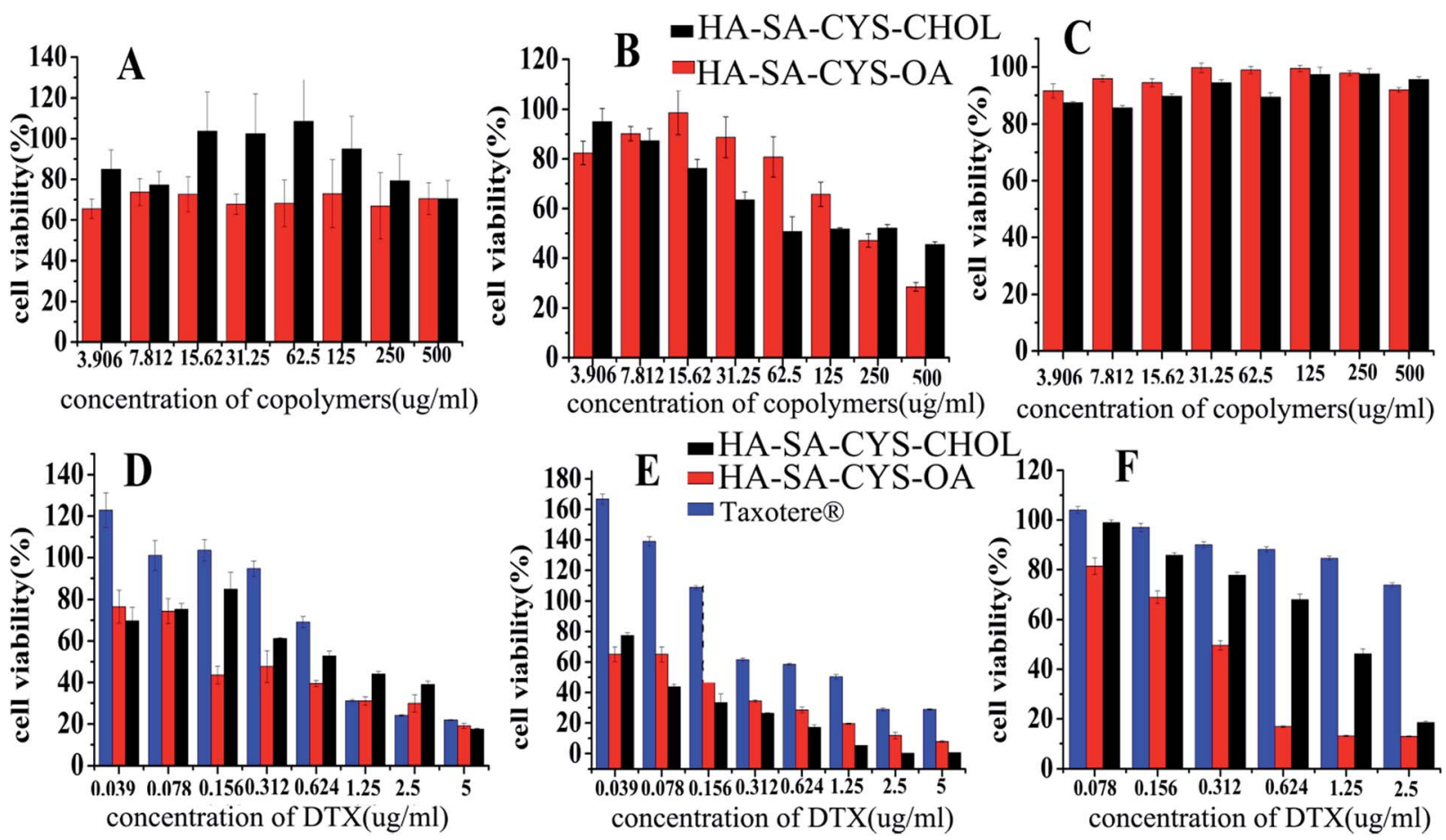

Fig. 5 In vitro cytotoxicity of (A) blank micelles in A549 cells, (B) blank micelles in MCF-7 cells, (C) blank micelles in S-180 cells, (D) DTX-loaded micelles and Taxotere $\AA$ in A549 cells, (E) DTX-loaded micelles and Taxotere $®$ in MCF-7 cells and (F) DTX-loaded micelles and Taxotere ${ }^{\circledR}$ in S180 cells (mean \pm S.D, $n=3$ ).

Table $2 \quad I C_{50}$ values of different DTX formulations against carcinomatous

\begin{tabular}{lccr}
\hline Cell line & Taxotere ${ }^{\circledR}\left(\mathrm{ng} \mathrm{mL}^{-1}\right)$ & ${\text { HA-SA-CYS-CHOL }\left(\mathrm{ng} \mathrm{mL}^{-1}\right)}^{\text {HA-SACYS-OA }\left(\mathrm{ng} \mathrm{mL}^{-1}\right)}$ \\
\hline A549 & $1191.0 \pm 76.825$ & $175 \pm 51.84$ & $118 \pm 3.72$ \\
MCF-7 & $607.4 \pm 42.8$ & $43 \pm 3.11$ & $10.8 \pm 1.26$ \\
S-180 & $14613.9 \pm 556.8$ & $1865.8 \pm 92.56$ & $254.3 \pm 31.63$
\end{tabular}


particle size. It was reported previously that octadecanoic acid produced an inhibition of WRL, MCF-7, COLO cell lines ${ }^{56}$ and ECV304 human endothelial cells. ${ }^{51,57}$

Based on the above, we hypothesized that, compared with cholesterol; octadecanoic acid with a greater toxicity was more cytotoxic to A549, MCF-7 and S-180 cells, which is worth our further study on the mechanism of hydrophobic groups on the in vitro cytotoxicity.

In order to better and clear study the role of hydrophobic groups on antitumor effects, we selected S-180 cells for in vivo experiments because blank micelles were substantially noncytotoxic to the S-180 cell.

\section{In vitro cellular uptake}

As shown in CLSM (Fig. 6), it was clear that the intracellular fluorescence intensity of HA-SA-CYS-CHLO (OA) NPs in A549, MCF-7 and S-180 cells was significantly higher than that of free Cou6, which might be due to the endocytosis mediated by CD44 receptors. In A549 and S-180 cells, the amount of cellular uptake of Cou6 in HA-SA-CYS-CHOL and HA-SA-CYS-OA micelles was 28.94-, 26.48- and 25.56-, 23.42-fold higher than that of free Cou6, respectively. However, the intracellular uptake of HA-SACYS-OA micelles was significantly increased in MCF-7 cells, which was 4.53 -fold greater than that of HA-SA-CYS-CHOL micelles.

As seen in Fig. 7, pre-treating the CD44 $4^{+}$cancer cells (MCF-7, A549 and S-180 cells) with free HA significantly reduced the internalization of HA-SA-CYS-CHOL (OA) NPs. Only a weak fluorescence signal was observed, indicating that the free HA competitively bound to CD44 and inhibited the uptake of HA-
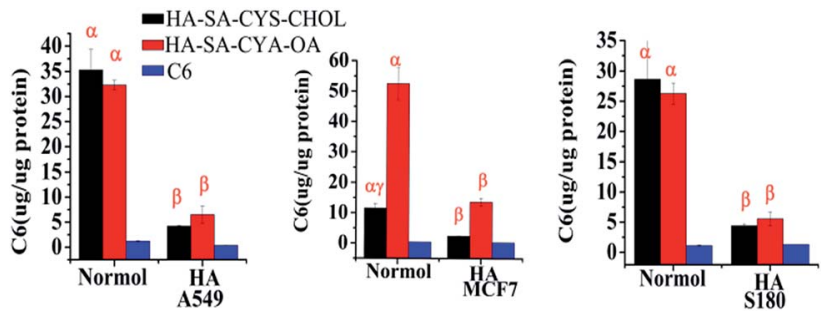

Fig. 7 Quantitative study of cellular uptake obtained from the experiments of cell uptake mechanism including the competitive inhibition study using $\mathrm{HA}$ solution (10 $\mathrm{mg} \mathrm{mL}^{-1}$ ). Bar graph data represent mean \pm SEM (95\% confidence interval), $n=6 . \alpha$ means NPs versus free cou6, $\beta$ means normal versus incubation with $H A, \gamma$ means $\mathrm{HA}-\mathrm{SA}-\mathrm{CYS}-\mathrm{CHOL}$ versus $\mathrm{HA}-\mathrm{SA}-\mathrm{CYS}-\mathrm{CHOL}(P<0.01)$.

SA-CYS-CHOL (OA) micelles. Combining the results of CLSM and quantitative analysis, we can conclude that the cellular uptakes of micelles are due to CD44 receptor-mediated endocytosis. And CD44-mediated endocytosis can effectively increase the uptake of NPs by tumour cells, which increasing the antitumour effect of the NPs.

\section{In vivo bio-distribution and tumour targeting}

As shown in Fig. 8A, in the experiments, most of the fluorescence was detected in the liver and spleen of DiR solution, indicating the DiR solution was rapid metabolized and excreted by hepatobiliary. Compared with DiR solution, HA-SA-CYSCHOL (OA) micelles exhibited a much stronger fluorescence signal in the tumour, which could be due to the HA located on the micelle surface increased the tumour uptake of micelles

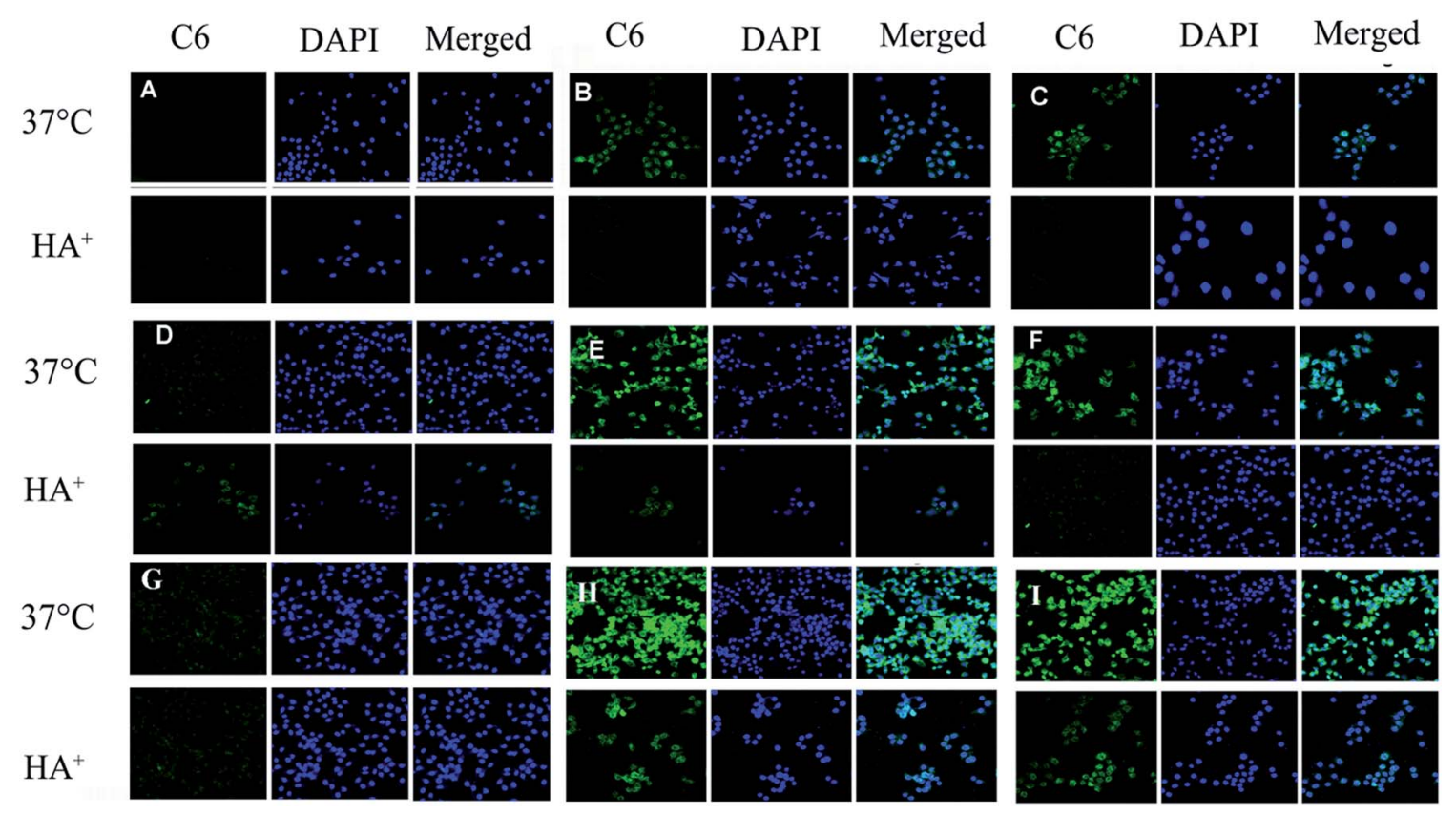

Fig. 6 Micelles enhance cellular uptake of the Cou6 into MCF-7, A549 and S-180 cell lines. Qualitative study of cellular uptake by confocal laser scanning microscope (CLSM) after $2 \mathrm{~h}$ incubation at $37 \mathrm{C}$ with (A) $3 \mu \mathrm{g} \mathrm{mL} \mathrm{L}^{-1}$ of free Cou6 in A549 cells, (B) HA-SA-CYS-CHOL NPs in A549 cells (C) HA-SA-CYS-OA NPs in A549 cells, (D) $3 \mu \mathrm{g} \mathrm{mL} \mathrm{L}^{-1}$ of free Cou6 in MCF-7 cells (E) HA-SA-CYS-CHOL NPs in MCF-7 cells, (F) HA-SA-CYSOA NPs in MCF-7 cells, (G) $3 \mu \mathrm{g} \mathrm{mL} \mathrm{m}^{-1}$ of free Cou6 in S-180, (H) HA-SA-CYS-CHOL NPs in S-180 cells, (I) HA-SA-CYS-OA NPs in S-180 cells. 


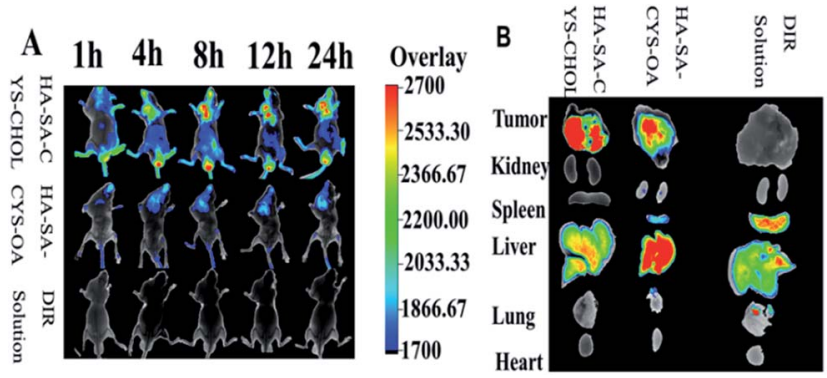

Fig. 8 In vivo disposition of DiR-loaded micelles in tumour-bearing mice. (A) NIRF images of DiR solution and HA-SA-CYS-CHOL, HASA-CYS-OA micelles at different study intervals. (B) Ex vivo fluorescence images of tissue samples collected $24 \mathrm{~h}$ post-injection.

through CD44 receptor and the EPR effect. ${ }^{58} \mathrm{~A}$ significant fluorescence signal could be detected as early as $1 \mathrm{~h}$ after injection and peaked at $24 \mathrm{~h}$ of both micelles. In the all times, the HA-SACYS-CHOL micelles showed much more stronger and lasting accumulation in cancer cells compared with HA-SA-CYS-OA micelles. As shown in Fig. 8B, the excised tumours' fluorescent image further confirmed the results observed in vivo. The HASA-CYS-CHOL micelles displayed higher fluorescence signal in cancer cells than the HA-SA-CYS-OA micelles and free DiR. Compared with HA-SA-CYS-CHOL micelles, much more micelles accumulated in the liver, kidney and spleen for the HA-SA-CYS-OA micelles, especially in liver, which indicating the rapid metabolism and excretion of HA-SA-CYS-OA micelles in vivo. However, for the HA-SA-CYS-CHOL micelles, most of the micelles were distributed in the tumour tissue, and only a small proportion of the micelles were metabolized. The difference between the two amphiphilic carriers was merely the hydrophobic group. Compared with octadecanoic acid, hyaluronic acid and cholesterol are the necessary components of cytoplasmic matrix and cell membrane. After intravenous injection, endogenous substances prepared micelles can reduce the identification of immune system and reticuloendothelial system, minimized the metabolism and excretion of micelles, improved in the prolonged-circulation and tumour-targeting ability. For the fast-growing cancer cells, the micelles were like sugar-coated shells, tumour cells can uptake the micelles through CD44-mediated receptor endocytosis for the proliferation. However, octadecanoic acid, as an exogenous material, will be identified, metabolized and excreted out of the body even though the hydrophobic group was in the core of the micelles. Therefore, we concluded that the hydrophobic groups affected the distribution, metabolism, and excretion of micelles.

\section{In vivo antitumor efficacy}

The in vivo antitumor efficacy verified the in vivo biodistribution and tumour targeting study. Significant decrease in tumour volume was detected in HA-SA-CYS-CHOL (OA) micelles compared with the control group after the third administration (Fig. 9A). HA-SA-CYS-CHOL (OA) micelles showed improved tumour suppression in comparison with
Taxotere ${ }^{\circledR}$ after the first administration, attributed to EPR effects and CD44 targeted cell uptake. At the end of the test, HASA-CYS-CHOL micelles had the lowest average tumour weight and tumour volume $(P<0.05)$. In vitro cytotoxicity experiments confirmed that hydrophobic groups were no cytotoxic to S-180 cells. From in vivo bio-distribution and tumour targeting study we have known that hydrophobic groups affected the distribution, metabolism, and excretion of micelles. Ultimately, the hydrophobic group will affect the anti-tumour effect of the micelles. So we assumed that the micelles do not change the molecular mechanism and signal pathways of drugs, it just traffics a greater fraction of the administered drug directly to cancer cells. And the anti-tumour signal pathways of micelles is that, DTX inhibits G2/M progression through destabilization of the microtubule network, which is required for spindle formation during mitosis, and increases the degradation of HIF- $1 \alpha$ by DTX induced-JNK2/PHD1 signaling pathway. ${ }^{59,60}$

The TIR of the HA-SA-CYS-CHOL and HA-SA-CYS-OA group, respectively, was $87.72 \%$ and $78.02 \%$ of the saline group. The isolated tumour average weight and size of the micelles were the smallest among the saline and Taxotere ${ }^{\circledR}$, further verifying that the DTX-loaded micelles had a superior in vivo therapeutic efficacy. More importantly, in this study, the administered dose was very low $\left(2 \mathrm{mg} \mathrm{kg}^{-1}\right)^{20}$ but we also achieved a pronounced antitumor effect.

In order to study the safety of the preparations, we monitored the body weight changes of mice for 15 days (Fig. 9B) and the net body weight variations on the sixteenth day were evaluated (Fig. 9C). Animals in the micelle groups, saline or Taxotere ${ }^{\circledR}$ groups did not show any weight loss during the entire study which reflected the reduced systemic toxicity of the given formulation.
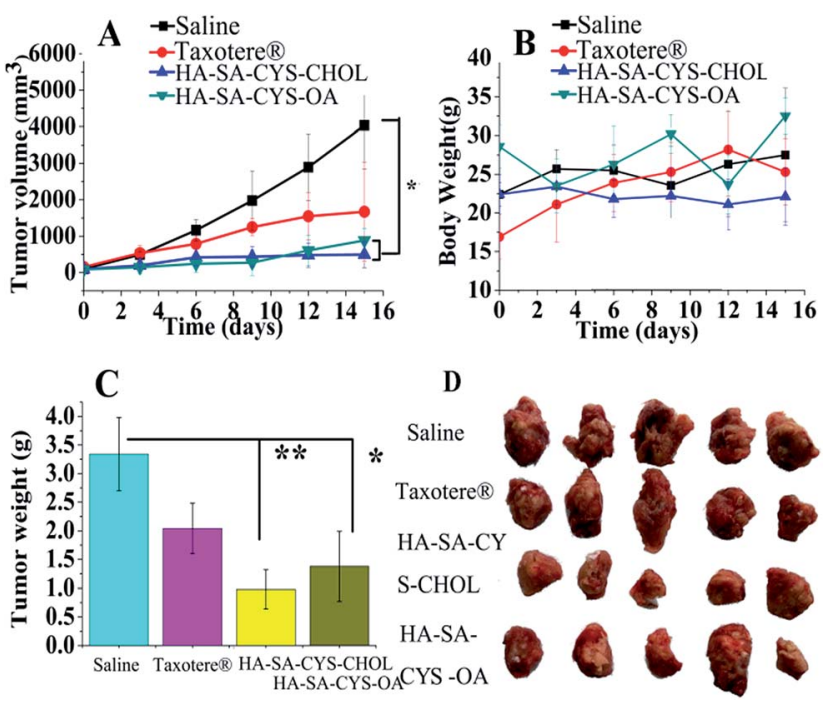

Fig. 9 Administration of micelles inhibited tumour growth. A: tumour volumes ( $n=5$ for each group). B: the body weights of mice ( $n=5$ for each group). C: the weights of tumours were calculated at time of sacrifice. D: the photograph of the tumours after biopsy. Bar graph data represent mean \pm SEM (95\% confidence interval), $n=5$ and $* P<$ $0.05, * * P<0.01$ versus saline. 


\section{Conclusion}

In this study, we successfully synthesized two kinds of amphiphilic copolymers with the same hydrophilic group to explore whether the different hydrophobic groups have an impact on the nature of the amphiphilic carriers and the micelles. From the experiments, we found that the different properties of hydrophobic groups of the amphiphilic carrier can affect the stability and drug-loading capacity of the amphiphilic carrier and the micelles. Compared with HA-SA-CYS-OA, HA-SA-CYSCHOL had a lower CMC contributing to the micelles having a smaller particle size and much higher encapsulation efficiency (EE) and drug loading (DL). However, HA-SA-CYS-OA had a greater EE when loaded withCou6, suggesting that the amphiphilic carrier had certain selectivity for hydrophobic drugs due to the effect of the different hydrophobic groups. Compared with HA-SA-CYS-CHOL, HA-SA-CYS-OA micelles displayed markedly higher cytotoxicity to MCF-7, A549 and S180 cells which may be due to the high toxicity of octadecanoic acid. The in vivo animal experiments confirmed that hydrophobic groups affected the distribution, metabolism, excretion and anti-tumour efficacy of micelles. HA-SA-CYSCHOL micelles possessed excellent tumour-targeting properties and efficient antitumor effects at very low concentrations with extremely low systemic toxicity. Therefore, we strongly recommend that when we examine amphiphilic polymer for drug delivery systems we carefully consider the interactions between hydrophobic groups and the drug loading and select the most appropriate hydrophobic groups to encapsulate the drugs. And we suggested that amphiphilic carrier should be synthesized from endogenous materials, if possible, because not only could it reduce cytotoxicity but also increase the antitumor effects. In the next experiment, we will further quantitatively study the effect of hydrophobic groups on the carriers and micelles.

\section{Acknowledgements}

This research has been under the support of the National Natural Science Foundation of China (No. 81273447).

\section{References}

1 S. Jaracz, J. Chen, L. V. Kuznetsova and I. Ojima, Bioorg. Med. Chem., 2005, 13, 5043-5054.

2 A. H. Kazunori Kataoka and Y. Nagasaki, Adv. Drug Delivery Rev., 2001, 2001, 113-131.

3 M. Z. Elsabee, R. E. Morsi and A. M. Al-Sabagh, Colloids Surf., $B, 2009$, 74, 1-16.

4 V. Wintgens and C. Amiel, Macromol. Chem. Phys., 2008, 209, 1553-1563.

5 Y. Y. Xiaofei Yuan, A. Harada and K. Kataoka, Langmuir, 2005, 2005, 2668-2674.

6 S. Y. Kuwahara, K. Yamamoto and J. I. Kadokawa, Chem. Lett., 2010, 39, 1045-1047.

7 A. Pinazo, V. Petrizelli, M. Bustelo, R. Pons, M. P. Vinardell, M. Mitjans, A. Manresa and L. Perez, Colloids Surf., B, 2016, 141, 19-27.
8 Y. Cheng, C. He, C. Xiao, J. Ding, X. Zhuang, Y. Huang and X. Chen, Biomacromolecules, 2012, 13, 2053-2059.

9 L. Prochazka, R. Tesarik and J. Turanek, Cell. Signalling, 2014, 26, 2234-2239.

10 Y. Yan, X. Zuo and D. Wei, Stem Cells Transl. Med., 2015, 4, 1033-1043.

11 V. Orian-Rousseau, Eur. J. Cancer, 2010, 46, 1271-1277.

12 M. P. Carvalho, E. C. Costa, S. P. Miguel and I. J. Correia, Carbohydr. Polym., 2016, 150, 139-148.

13 G. Tripodo, A. Trapani, M. L. Torre, G. Giammona, G. Trapani and D. Mandracchia, Eur. J. Pharm. Biopharm., 2015, 97, 400-416.

14 L. Mayol, M. Biondi, L. Russo, B. M. Malle, K. SchwachAbdellaoui and A. Borzacchiello, Carbohydr. Polym., 2014, 102, 110-116.

15 J. Li, T. Yin, L. Wang, L. Yin, J. Zhou and M. Huo, Int. J. Pharm., 2015, 483, 38-48.

16 Y. Liu, J. Sun, W. Cao, J. Yang, H. Lian, X. Li, Y. Sun, Y. Wang, S. Wang and Z. He, Int. J. Pharm., 2011, 421, 160-169.

17 A. Ritwiset, S. Krongsuk and J. R. Johns, Appl. Surf. Sci., 2016, 380, 23-31.

18 J. R. Madan, N. P. Ghuge and K. Dua, Drug Delivery Transl. Res., 2016, 6, 511-518.

19 X. Wei, T. H. Senanayake, G. Warren and S. V. Vinogradov, Bioconjugate Chem., 2013, 24, 658-668.

20 H. Liu, K. Li, L. Lan, J. Ma, Y. Zeng, L. Xu and D. Wu, J. Mater. Chem. B, 2014, 2, 5238-5248.

21 H. Liu, K. Li, L. Xu and D. Wu, J. Nanopart. Res., 2014, 16, 2784.

22 L. H. Wang, S. G. Ding, J. J. Yan and Y. Z. You, J. Phys. Chem. $B, 2014$, 118, 3893-3898.

23 X. Wang, J. T. Chen, H. Zhu, X. Chen and X. P. Yan, Anal. Chem., 2013, 85, 10225-10231.

24 Z. A. Hamid, A. Blencowe, B. Ozcelik, J. A. Palmer, G. W. Stevens, K. M. Abberton, W. A. Morrison, A. J. Penington and G. G. Qiao, Biomaterials, 2010, 31, 6454-6467.

25 M. Kong, H. Park, C. Feng, L. Hou, X. Cheng and X. Chen, Carbohydr. Polym., 2013, 94, 634-641.

26 J. Yang, Y. Huang, C. Gao, M. Liu and X. Zhang, Colloids Surf., B, 2014, 115, 368-376.

27 J. Li, M. Huo, J. Wang, J. Zhou, J. M. Mohammad, Y. Zhang, Q. Zhu, A. Y. Waddad and Q. Zhang, Biomaterials, 2012, 33, 2310-2320.

28 T. Yin, L. Wang, L. Yin, J. Zhou and M. Huo, Biomaterials, 2015, 61, 10-25.

29 J. Li, D. Liu, G. Tan, Z. Zhao, X. Yang and W. Pan, Carbohydr. Polym., 2016, 146, 435-444.

30 X. Zhang, F. Du, J. Huang, W. Lu, S. Liu and J. Yu, Colloids Surf., B, 2012, 100, 155-162.

31 R. Ran, Y. Liu, H. Gao, Q. Kuang, Q. Zhang, J. Tang, H. Fu, Z. Zhang and Q. He, J. Pharm. Sci., 2015, 104, 476-484.

32 S. Song, H. Qi, J. Xu, P. Guo, F. Chen, F. Li, X. Yang, N. Sheng, Y. Wu and W. Pan, Pharm. Res., 2014, 31, 2988-3005.

33 H. K. Park, S. J. Lee, J. S. Oh, S. G. Lee, Y. I. Jeong and H. C. Lee, Nanoscale Res. Lett., 2015, 10, 981. 
34 A. Martinez, E. Muniz, C. Teijon, I. Iglesias, J. M. Teijon and M. D. Blanco, Pharm. Res., 2014, 31, 1264-1274.

35 S. K. Pandey, D. K. Patel, A. K. Maurya, R. Thakur, D. P. Mishra, M. Vinayak, C. Haldar and P. Maiti, Int. J. Biol. Macromol., 2016, 89, 99-110.

36 L. Qiu, Z. Li, M. Qiao, M. Long, M. Wang, X. Zhang, C. Tian and D. Chen, Acta Biomater., 2014, 10, 2024-2035.

37 S. Yin, J. Huai, X. Chen, Y. Yang, X. Zhang, Y. Gan, G. Wang, X. Gu and J. Li, Acta Biomater., 2015, 26, 274-285.

38 E. Giovannetti, V. Mey, S. Nannizzi, G. Pasqualetti, L. Marini, M. Del Tacca and R. Danesi, Mol. Pharmacol., 2005, 68, 110118.

39 J. R. Tonra, D. S. Deevi, E. Corcoran, H. Li, S. Wang, F. E. Carrick and D. J. Hicklin, Clin. Cancer Res., 2006, 12, 2197-2207.

40 T. C. Chou, Pharmacol. Rev., 2006, 58, 621-681.

41 F. Q. Hu, X. L. Wu, Y. Z. Du, J. You and H. Yuan, Eur. J. Pharm. Biopharm., 2008, 69, 117-125.

42 M. A. Ward and T. K. Georgiou, Polym. Chem., 2013, 4, 1893. 43 D. Xin, Y. Wang and J. Xiang, Pharm. Res., 2009, 27, 380-389. 44 A. K. Yadav, A. Agarwal, G. Rai, P. Mishra, S. Jain, A. K. Mishra, H. Agrawal and G. P. Agrawal, Drug Delivery, 2010, 17, 561-572.

45 J. M. Brown, Cancer Biol. Ther., 2014, 1, 453-458.

46 D. Hanahan and R. A. Weinberg, Cell, 2011, 144, 646-674.

47 A. G. Cuenca, H. Jiang, S. N. Hochwald, M. Delano, W. G. Cance and S. R. Grobmyer, Cancer, 2006, 107, 459-466.
48 S. Bhattacharjee, J. Controlled Release, 2016, 235, 337-351. 49 G. Saravanakumar, K. Y. Choi, H. Y. Yoon, K. Kim, J. H. Park, I. C. Kwon and K. Park, Int. J. Pharm., 2010, 394, 154-161.

50 Q.-M. L. Dong Ma, Li-M. Zhang, Y.-Y. Liang and W. Xue, Biomaterials, 2014, 2014, 4357-4367.

51 B. Atasever-Arslan, K. Yilancioglu, M. G. Bekaroglu, E. Taskin, E. Altinoz and S. Cetiner, Gen. Physiol. Biophys., 2015, 34, 201-207.

52 G. R. Tan, S. S. Feng and D. T. Leong, Biomaterials, 2014, 35, 3044-3051.

53 X. C. Bin Du, F. Zhao, X. Su, Y. Wang, X. Yan, S. Jia, J. Zhou and H. Yao, J. Mater. Chem. B, 2016, 2016, 2038-2050.

54 N. Basakran, Saudi Med. J., 2015, 36, 273-279.

55 R. Thapa and G. D. Wilson, Stem Cells Int., 2016, 2016, 2087204.

56 O. O. Aiyelaagbe, A. S. Negi, A. A. Hamid, S. Luqman, S. B. Kumar and F. Kaneez, J. Chin. Chem. Soc., 2015, 62, 751-755.

57 J. Ji, L. Zhang, P. Wang, Y. M. Mu, X. Y. Zhu, Y. Y. Wu, H. Yu, B. Zhang, S. M. Chen and X. Z. Sun, Exp. Toxicol. Pathol., 2005, 56, 369-376.

58 Y. Nakamura, A. Mochida, P. L. Choyke and H. Kobayashi, Bioconjugate Chem., 2016, 27, 2225-2238.

59 P. J. M. A. F. A. Fitzpatrick, Proc. Natl. Acad. Sci. U. S. A., 1998, 95, 3896-3901.

60 E. T. Oh, C. W. Kim, S. J. Kim, J. S. Lee, S. S. Hong and H. J. Park, Sci. Rep., 2016, 6, 27382. 